\title{
Efficacy and Tolerability of Intravenous Ferric Carboxymaltose in Patients with Iron Deficiency at a Hospital Outpatient Clinic: A Retrospective Cohort Study of Real-World Clinical Practice
}

\author{
António Robalo Nunes, ${ }^{1}$ Ana Palricas Costa, ${ }^{2}$ \\ Sara Lemos Rocha, ${ }^{2}$ and Ana Garcia de Oliveira ${ }^{2}$ \\ ${ }^{1}$ Hospital de Dia de Imuno-Hemoterapia, Hospital Pulido Valente, Centro Hospitalar Lisboa Norte (CHLN), \\ Alameda das Linhas de Torres 117, 1769-001 Lisboa, Portugal \\ ${ }^{2}$ Hospital de Dia de Imuno-Hemoterapia, Hospital de Santa Maria, CHLN, Avenida Prof. Egas Moniz, 1649-035 Lisboa, Portugal \\ Correspondence should be addressed to António Robalo Nunes; robalonunes@gmail.com
}

Received 17 March 2017; Revised 29 May 2017; Accepted 1 June 2017; Published 3 July 2017

Academic Editor: Ajit C. Gorakshakar

Copyright @ 2017 António Robalo Nunes et al. This is an open access article distributed under the Creative Commons Attribution License, which permits unrestricted use, distribution, and reproduction in any medium, provided the original work is properly cited.

Ferric carboxymaltose (FCM) is an intravenous iron formulation to correct iron deficiency. Although its use has been extensively studied in clinical trials, real-world evidence regarding FCM treatment is scarce. Our aim was to evaluate the efficacy and tolerability of FCM treatment in patients with iron deficiency, with or without anemia, at a hospital outpatient clinic. Data was collected retrospectively from medical records. During this 2-year study, 459 patients were included. Mean age was $58.6 \pm 17.5$ years and most patients received cumulative FCM doses of 501-1000 mg (63.2\%). Six weeks after administration of FCM, efficacy endpoints hemoglobin increase $\geq 2 \mathrm{~g} / \mathrm{dL}$, hemoglobin increase $\geq 3 \mathrm{~g} / \mathrm{dL}$, and transferrin saturation $>20 \%$ were attained by $41 \%, 20 \%$, and $63 \%$ of patients, respectively. Patients who received higher FCM doses showed significant reduced odds of not achieving hemoglobin increase $\geq 2 \mathrm{~g} / \mathrm{dL}$ (501-1000 mg, adjusted odds ratio [OR]: 0.34, 95\% confidence interval [CI] 0.18-0.62; 1001-3000 mg, OR: 0.19, 95\% CI 0.07-0.49), compared to $500 \mathrm{mg}$ doses. Treatment-emergent adverse events were documented in $<4 \%$ of patients. In conclusion, FCM treatment was effective and well-tolerated by outpatients with iron deficiency at a hospital clinic, and its dosage should be adjusted to improve iron deficiency management in clinical practice.

\section{Introduction}

Iron deficiency is the most common nutritional deficiency worldwide. It has been estimated to affect at least $30-40 \%$ of preschool children and pregnant women and about one-sixth of the total population in developed countries [1-3]. Iron deficiency is frequently associated with fatigue, susceptibility to infections, and poorer work capacity and quality of life and is a major cause for anemia in both developing and developed countries [1,2]. Moreover, comorbidity of iron deficiency and anemia with other diseases have been described to increase the risk of morbidity and mortality $[2,4]$.

Iron deficiency can occur in two main types: absolute, associated with low total body iron stores due to decreased dietary intake or blood loss, or functional, which occurs when the iron absorption or release from storage areas is inadequate, despite normal or increased total body iron stores [4-6]. Functional iron deficiency may be caused by chronic inflammation, associated with several conditions such as renal failure, congestive heart failure, inflammatory bowel disease, cancer, or autoimmune conditions, which contributes to the onset of the anemia of chronic diseases $[7,8]$.

Treatment for iron deficiency involves the restoration of iron stores to normal levels and the increase of hemoglobin values, as well as addressing its underlying causes [9]. Oral iron supplementation is the first-line therapy for iron deficiency [6]. However, oral iron therapy presents disadvantages, such as low absorption of iron, drug interactions, increased oxidative stress in the gastrointestinal tract, or 
high incidence of gastrointestinal adverse events, which can reduce patient compliance [4]. Thus, oral iron has limited efficacy in treating chronic iron deficiency anemia (IDA), hence being more adequate for short periods of iron requirements $[6,10]$. Intravenous iron formulations such as ferric carboxymaltose, iron dextran, ferric gluconate, and iron sucrose may potentially solve these issues and may be more convenient both to healthcare providers and patients, if oral iron therapy does not provide the required correction of iron deficiency or is not well-tolerated by patients $[4,6,9]$.

Ferric carboxymaltose (FCM) is a relatively recent intravenous iron formulation, which can be administered in short periods of time and at large single doses (up to $1000 \mathrm{mg}$ ) $[4,11]$. Its efficacy in correcting iron deficiency has been evaluated in several adult populations, namely, patients with gastrointestinal disorders, chronic kidney disease, chronic heart failure, gynecological and obstetrics disorders, and neoplasms $[4,5,7-10,12]$. Indeed, these studies highlighted the advantages of using intravenous FCM in detriment of oral iron, in terms of both efficacy and tolerability $[4,8-10,12]$.

In clinical trials, FCM was associated with rapid hematopoietic improvement by increasing hemoglobin levels, refurnishing iron stores (i.e., changes in ferritin levels), and increasing available iron for erythropoiesis (i.e., raise in transferrin saturation) $[9,12]$. Administration of FCM was also well-tolerated by patients, with most drugrelated adverse events considered to be mild to moderate in severity $[4,10,12]$. However, real-world data on the efficacy of intravenous FCM therapeutics in current clinical practice are still scarce $[5,8,13,14]$. Therefore, the purpose of this study was to evaluate the efficacy and tolerability of intravenous FCM treatment of iron deficiency, in patients with or without anemia, attending an outpatient hospital clinic setting.

\section{Materials and Methods}

2.1. Study Design. This was a retrospective cohort study, conducted at the Immunohemotherapy (transfusion medicine) outpatient clinic of Hospital Pulido Valente and Hospital de Santa Maria, both hospitals of Centro Hospitalar Lisboa Norte. Data was collected retrospectively from medical records on all consecutive patients with iron deficiency attending the hospital outpatient clinic, who were treated with intravenous FCM from January 2014 to December 2015. For each patient included in this study, data was only collected regarding treatment of first episode with a follow-up period of six weeks ( \pm 1 week), and no subsequent episodes were included.

2.2. Patients. Eligible patients were adult individuals $(\geq 18$ years old) who had iron deficiency with or without anemia and were submitted to intravenous FCM therapeutics at the hospital outpatient clinic. Iron deficiency was defined as transferrin saturation $<20 \%$ [15]. Anemia was defined as hemoglobin $\leq 12 \mathrm{~g} / \mathrm{dL}$ in women, and $\leq 13 \mathrm{~g} / \mathrm{dL}$ in men, according to the World Health Organization threshold hemoglobin concentrations [1]. Patients were excluded if they had missing data regarding any of the hematological or iron laboratory tests or did not complete the first episode treatment with FCM.

Patients were assigned to FCM dose levels according to their individual potential iron requirement, as recommended by the Summary of Product Characteristics (SmPC) or calculated using the Ganzoni formula [16]. According to this formula, individual potential iron requirement is a function of the patient's hemoglobin level and body weight: total iron deficit $(\mathrm{mg})=$ body weight $(\mathrm{kg}) \times($ target hemoglobin - actual hemoglobin $)(\mathrm{g} / \mathrm{dL}) \times 2.4+$ depot iron of $500 \mathrm{mg}$.

Patients received single doses of FCM from $500 \mathrm{mg}$ or $15 \mathrm{mg} / \mathrm{kg}$ up to a maximum dose of $1000 \mathrm{mg}$ per week, given as intravenous infusions over 15 to $30 \mathrm{~min}$.

2.3. Data Collection. Demographic, clinical, laboratory, and safety data were retrospectively collected from patients' medical records, at admission (before treatment) and followup visits (after treatment). Demographic data included age and gender, while clinical data included primary diagnosis, cumulative iron dose levels, and adverse events. Primary diagnoses were coded using the high-level classification of the 10th revision of the International Statistical Classification of Diseases and Related Health Problems (ICD-10) [17]. Patients were stratified by cumulative iron dose levels in three categories: $500 \mathrm{mg}, 501-1000 \mathrm{mg}$, and 1001-3000 mg. Laboratory test results data included pretreatment and posttreatment serum hemoglobin, hematocrit, mean corpuscular volume $(\mathrm{MCV})$, mean corpuscular hemoglobin $(\mathrm{MCH})$, ferritin, and transferrin saturation. Reference ranges of the local laboratory were $12.0-15.3 \mathrm{~g} / \mathrm{dL}$ (female) or $13.0-17.5 \mathrm{~g} / \mathrm{dL}$ (male) for hemoglobin; $36-46 \%$ (female) or $40-50 \%$ (male) for hematocrit; 80-97 fL (female or male) for MCV; 27-33 pg (female or male) for $\mathrm{MCH}$; and $13-150 \mathrm{ng} / \mathrm{mL}$ (female) or 30-400 ng/mL (male) for ferritin.

2.4. Endpoints. The primary efficacy endpoint of the data analysis was the proportion of patients with a hemoglobin increase of $\geq 2.0 \mathrm{~g} / \mathrm{dL}$ after treatment (i.e., achieving a hematopoietic response). Secondary efficacy endpoints were the proportion of patients attaining a hemoglobin increase of $\geq 3.0 \mathrm{~g} / \mathrm{dL}$ after treatment, and the proportion of patients attaining a transferrin saturation $>20 \%$ after treatment (i.e., achieving an iron bioavailability response).

Safety endpoint was the incidence of treatment-emergent adverse events (TEAEs) occurring during or after administration of intravenous FCM. All safety data were collected during clinical care, without a systematic method, which means some data might not have been registered in the patient files.

2.5. Statistical Analysis. Continuous variables were summarized by mean and standard deviation or median and 1st quartile-3rd quartile as applicable. Categorical variables were summarized by relative and absolute frequencies. The variation in the hematological and iron parameters was assessed using the paired $t$-test or Wilcoxon signed-rank test as applicable.

Primary diagnoses were coded using the high-level classification of ICD-10. For the analyses of the efficacy endpoints, 
classes with less than 10 patients were merged into a single class named "other diseases," to ensure an adequate statistical power for the comparisons. The remaining classes (i.e., neoplasms, diseases of the genitourinary system, diseases of the circulatory system, and diseases of the digestive system) were analyzed individually.

Due to the study design, no reference group was established. Therefore, pairwise exploratory comparisons regarding diagnoses groups were performed against all the remaining subjects (e.g., the group of patients with neoplasms was compared against the group of subjects who did not have neoplasms). As for the dosage groups, pairwise exploratory comparisons were performed against the lower dose (i.e., $500 \mathrm{mg}$ ), used as reference dose.

To assess the treatment efficacy, a logistic regression model was used to determine the probability of clinical failure in each endpoint (i.e., not achieving the efficacy endpoint after treatment with intravenous FCM). The odds ratio (OR) and 95\% confidence intervals (CIs) were estimated. The computed OR were adjusted for age and pretreatment hemoglobin for the endpoints related to hemoglobin increase and for age and pretreatment transferrin saturation for the endpoint related to the increase of transferrin saturation.

Due to the low incidence of adverse events, no statistical inference was performed.

\section{Results}

3.1. Patients Characteristics. A total of 459 patients who received intravenous FCM treatment were included in the study. Demographic and clinical characteristics of the patients are shown in Table 1. Mean age was $58.6 \pm 17.5$ years and most patients were female (78\%). In all, anemia was recorded in $82.4 \%$ of patients, iron deficiency was recorded in $98 \%$ of patients, and IDA was recorded in $81.3 \%$ of patients. The majority of patients received a single FCM dose between $501 \mathrm{mg}$ and $1000 \mathrm{mg}$ (63.2\%). Only 10.2\% of patients received cumulative iron doses from $1001 \mathrm{mg}$ to $3000 \mathrm{mg}$ in divided doses (one per week). Most common primary diagnoses were diseases of the digestive system (43.4\%), diseases of the genitourinary system (26.4\%), neoplasms $(10.2 \%)$, and diseases of the circulatory system (8.3\%). Other diseases comprised the following categories: diseases of the blood and blood-forming organs and certain disorders involving the immune mechanism (3.3\%); endocrine, nutritional, and metabolic diseases $(2.0 \%)$; diseases of the musculoskeletal system and connective tissue (1.7\%); diseases of the nervous system (1.5\%); diseases of the eye and adnexa (1.3\%); pregnancy, childbirth, and the puerperium (1.1\%); diseases of the respiratory system $(0.7 \%)$; and certain infectious and parasitic diseases $(0.2 \%)$. After 6 weeks ( \pm 1 week) following FCM treatment, all hematological and iron measurements significantly increased from baseline (Table 2).

3.2. Efficacy Endpoints. The primary (i.e., hemoglobin increase $\geq 2 \mathrm{~g} / \mathrm{dL}$ ) and secondary (i.e., hemoglobin increase $\geq 3 \mathrm{~g} / \mathrm{dL}$ and transferrin saturation $>20 \%$ ) efficacy endpoints, following intravenous FCM treatment, are shown in Table 3.
TABLE 1: Demographic and clinical characteristics of the study population.

\begin{tabular}{lc}
\hline Characteristic & $\begin{array}{c}\text { Patients } \\
(N=459)\end{array}$ \\
\hline Age (years), mean (SD) & $58.6(17.5)$ \\
Elderly > 65 years, $n(\%)$ & $187(40.7)$ \\
Male, $n(\%)$ & $101(22.0)$ \\
Anemia, $n(\%)$ & $378(82.4)$ \\
Iron deficiency, $n$ (\%) & $450(98.0)$ \\
Iron deficiency anemia, $n(\%)$ & $373(81.3)$ \\
Iron deficiency without anemia, $n(\%)$ & $77(16.8)$ \\
Cumulative FCM treatment dose, $n(\%)$ & \\
500 mg & $122(26.6)$ \\
$501-1000$ mg & $290(63.2)$ \\
1001-3000 mg & $47(10.2)$ \\
Diagnosis per ICD-10 high level category, $n(\%)$ & \\
Diseases of the digestive system & $199(43.4)$ \\
Diseases of the genitourinary system & $121(26.4)$ \\
Neoplasms & $47(10.2)$ \\
Diseases of the circulatory system & $38(8.3)$ \\
Other diseases & $54(11.8)$ \\
\hline
\end{tabular}

FCM: ferric carboxymaltose; ICD-10: International Classification of Diseases 10th Revision.

After 6 weeks, hemoglobin increase of $\geq 2 \mathrm{~g} / \mathrm{dL}$ was attained by $41 \%$ of all patients, $40 \%$ in the IDA group, $40 \%$ in the diseases of the digestive system group, $55 \%$ in the diseases of the genitourinary system group, $26 \%$ in the neoplasms group, and $29 \%$ in the diseases of the circulatory system group. Moreover, our analysis indicated that patients with diseases of the genitourinary system presented significant decreased odds of clinical failure (OR: $0.46,95 \%$ CI $0.25,0.87$ ), whereas patients with diseases of the circulatory system showed about three times higher odds of clinical failure (OR: 3.34, 95\% CI 1.31-8.98). Regarding cumulative FCM treatment dose, we found significant decreased odds of clinical failure in patients who received doses ranging from 501 to $1000 \mathrm{mg}$ (OR: $0.34,95 \%$ CI $0.18-0.62$ ) or $1001-3000 \mathrm{mg}$ doses (OR: $0.19,95 \%$ CI $0.07-0.49$ ), compared to patients who received doses of $500 \mathrm{mg}$ (Table 3).

Hemoglobin increase of $\geq 3 \mathrm{~g} / \mathrm{dL}$ after 6 weeks after FCM dose was attained by $20 \%$ of all patients, $24 \%$ of the patients in the IDA group, $22 \%$ in the diseases of the digestive system group, $26 \%$ in the diseases of the genitourinary system group, $11 \%$ in the neoplasms group, and $16 \%$ in the diseases of the circulatory system group. Furthermore, our analysis indicated that patients with IDA presented significant lower odds of clinical failure (OR: 0.07, 95\% CI 0.01-0.24). Concerning total FCM treatment dose, our analysis showed significant lower odds of clinical failure in patients who received doses ranging from 501 to $1000 \mathrm{mg}$ (OR: $0.36,95 \% \mathrm{CI} 0.12-0.92$ ) or 1001-3000 mg doses (OR: $0.23,95 \%$ CI 0.06-0.73), compared to patients who received doses of $500 \mathrm{mg}$ (Table 3).

Finally, transferrin saturation $>20 \%$ after 6 weeks after FCM dose was attained by $63 \%$ of all patients, $69 \%$ of patients 
TABLE 2: Hematological laboratory tests before and after treatment with intravenous ferric carboxymaltose (FCM).

\begin{tabular}{lccr}
\hline Variables & Before treatment & After treatment & $p$ value \\
\hline Hemoglobin $(\mathrm{g} / \mathrm{dL})$ & $10.67(1.67)$ & $12.65(1.26)$ & $<0.001$ \\
Hematocrit $(\%)$ & $33.32(4.48)$ & $38.57(3.81)$ & $<0.001$ \\
MCV (fL) & $80.67(8.94)$ & $86.75(6.56)$ & $<0.001$ \\
MCH $(\mathrm{pg})$ & $25.62(3.63)$ & $28.30(2.37)$ & $<0.001$ \\
Ferritin $(\mu \mathrm{g} / \mathrm{dL})$ & $9.60(5.70-24.00)$ & $137.00(59.95-243.10)$ & $<0.001$ \\
Transferrin saturation $(\%)$ & $8.70(4.76)$ & $25.02(10.16)$ & $<0.001$ \\
\hline
\end{tabular}

Values are expressed as mean (standard deviation) or median (1st quartile-3rd quartile). Laboratory reference ranges in normal adults: hemoglobin, $12.0-15.3 \mathrm{~g} / \mathrm{dL}$ (female) and 13.0-17.5 g/dL (male); hematocrit, 36-46\% (female) and 40-50\% (male); MCV, 80-97 fL; MCH, 27-33 pg; ferritin, 13-150 $\mu \mathrm{g} / \mathrm{dL}$ (female) and 30-400 $\mu \mathrm{g} / \mathrm{dL}$ (male); MCH: mean corpuscular hemoglobin; MCV: mean corpuscular volume.

in both IDA and iron deficiency without anemia groups, $63 \%$ in the diseases of the digestive system group, $68 \%$ in the diseases of the genitourinary system group, $67 \%$ in the neoplasms group, and $47 \%$ in the diseases of the circulatory system group. Our analysis indicated significant lower odds of clinical failure in patients who received doses ranging from 501 to $1000 \mathrm{mg}$ (OR: $0.57,95 \%$ CI $0.36-0.88$ ) or $1001-3000 \mathrm{mg}$ doses (OR: $0.25,95 \%$ CI $0.10-0.55$ ), compared to patients who received doses of $500 \mathrm{mg}$ (Table 3 ).

3.3. Safety Endpoint. A total of 17 TEAEs were recorded in $15(3.3 \%)$ patients. The most common documented TEAEs were nausea $(n=4)$, rash $(n=4)$, and urticaria $(n=2)$. Additionally, patients also experienced diarrhea $(n=1)$, chill $(n=1)$, myalgia $(n=1)$, abdominal pain $(n=1)$, wheeze $(n=1)$, arthralgia $(n=1)$, and hyperthermia $(n=1)$.

\section{Discussion}

This study evaluated the efficacy and tolerability of intravenous FCM treatment for the correction of iron deficiency, with or without anemia, in patients with diverse diagnoses, who were attending a hospital outpatient Immunohemotherapy clinic. The rationale for studying the complete population of patients under FCM treatment and not only some subgroups as most studies report $[8,14,18-21]$ was to improve the prescription standards of the intravenous FCM in clinical practice.

Treatment efficacy with FCM considering the primary endpoint of hemoglobin increase $\geq 2 \mathrm{~g} / \mathrm{dL}$ at week 6 was $41 \%$ in all patients, in agreement with previous studies performed in various populations of patients with IDA $[4,5,8,9]$. For patients with IDA, no differences in treatment efficacy were found for hemoglobin increase of $\geq 2 \mathrm{~g} / \mathrm{dL}$ and transferrin saturation $>20 \%$, compared with patients without IDA. However, we found a significant difference in hemoglobin increase of $\geq 3 \mathrm{~g} / \mathrm{dL}$. This finding was expected, as achieving such a high increase is more challenging for patients with normal hemoglobin levels.

Efficacy of FCM was lower in patients with neoplasms and diseases of the circulatory system. This might be due to the increased inflammatory states commonly caused by these diseases (e.g., cancer, acute or chronic congestive heart failure), which are known to impair patients' ability to absorb iron and hence to achieve a rapid hemoglobin increase [8]. To illustrate, neoplasms patients achieved lower efficacy in hemoglobin increase, but higher efficacy in transferrin saturation $>20 \%$. This finding is consistent with the presence of anemia of chronic disease rather than absolute iron deficiency (i.e., low body iron stores), which makes these patients less respondent to iron supplementation $[7,8,11,22]$. In contrast, FCM efficacy was significantly higher in patients with diseases of the genitourinary system for hemoglobin increase, as most of the diagnoses included in this group were heavy uterine bleeding, a condition that presents a good response to iron treatment due to its pathophysiology $[5,8$, $10,21,22]$. Accordingly, male patients presented an apparent lower efficacy rate, although not significant. Finally, efficacy of FCM treatment in patients with diseases of the digestive system was comparable to the ones of other subgroups. This finding is consistent with the pathophysiology of most diseases in this subgroup, which include either food disorders or acute bleedings. Other diseases had a low presence to allow any conclusion.

The incidence of TEAEs was low in this study $(<4 \%)$. Rash and nausea were the most common TEAEs, and no serious adverse events were found in the medical records. Although these events might be underreported, it was not expected that FCM would present a high toxicity, as other studies also reported FCM as a well-tolerated therapy [6, 9-12, 23, 24] with an acceptable safety profile in patients with diverse diagnoses $[5,8,10,11,23]$. Nevertheless, the conservativeness in the cumulative FCM treatment doses might also be a factor that explains the low incidence of TEAEs.

Not so surprisingly, higher cumulative doses of FCM (i.e., $501-1000 \mathrm{mg}$ and $1001-3000 \mathrm{mg}$ ) showed a significant higher efficacy compared to the lower dose (i.e., $500 \mathrm{mg}$ ), used as reference. In this study, doses of $500 \mathrm{mg}$ were on average $75 \%$ less effective than doses above $1000 \mathrm{mg}$, even when adjusted for age and predose hemoglobin or transferrin saturation serum levels. Although no data on body weight was collected, we may assume that patients were frequently treated with lower doses than the ones recommended in the SmPC due to the high frequency of cumulative FCM doses of $500 \mathrm{mg}$ (26.6\%). This lower dosage profile might affect overall treatment efficacy in our clinical setting, as demonstrated by the lower efficacy rates associated with lower cumulative FCM doses shown in this study. 


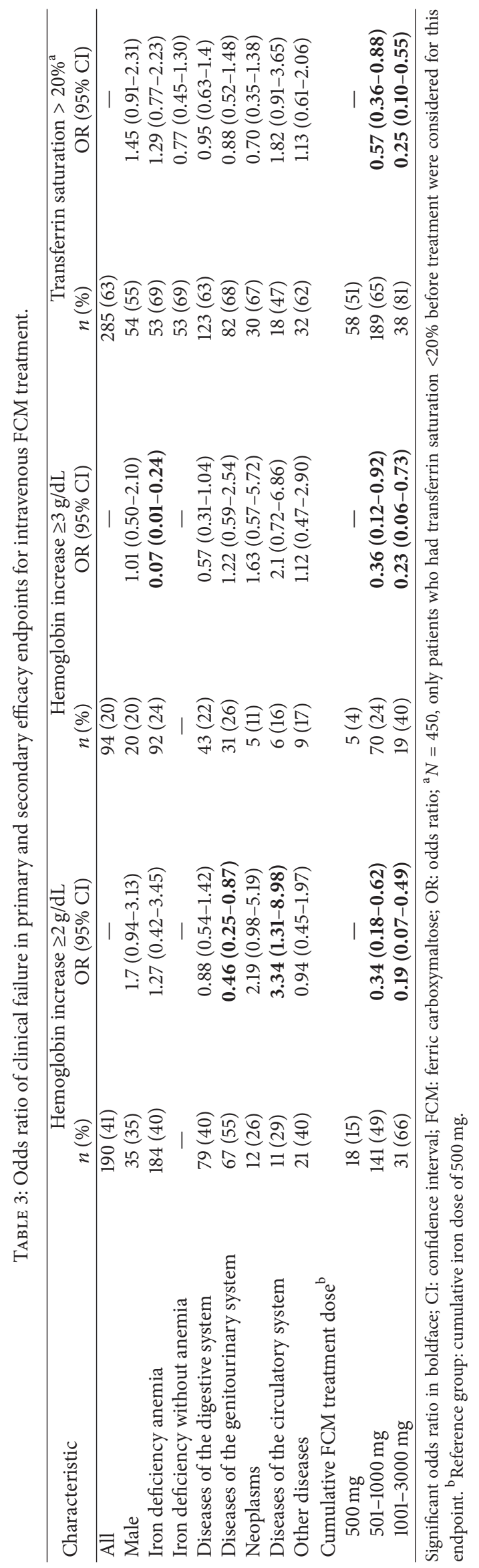


Although most clinical trials and studies reported low toxicity for FCM, clinicians might be still using the traditionally lower doses of oral iron therapy, which is less tolerated by the patients $[6,9-12,23,24]$. Therefore, our results suggest that patients might be undertreated due to treatment conservativeness of the clinicians prescribing intravenous iron therapeutics. Indeed, we found better hematological responses with the use of higher cumulative FCM doses. Hence, our study provides additional data supporting the indication of higher cumulative FCM doses for the treatment of iron deficiency, across patients with diverse diagnoses. As previously reported by other authors $[10,18,22,23,25]$, higher FCM doses may benefit the patients in terms of treatment efficacy and quality of life, without significantly impacting treatment tolerability and safety. In addition, administration of single large doses up to $1000 \mathrm{mg}$, instead of multiple lower iron dosages, is more convenient for the patients and may result in cost savings for healthcare systems and society [5, 8].

To our knowledge, this is one of the first observational studies reporting the efficacy of intravenous FCM treatment in a real-world setting, across a population of patients with a considerable dimension $(n=459)[5,8,13,14]$. Moreover, this study presents data on a single drug formulation, prescribed to patients with diverse ages and primary diagnoses. Therefore, we may assume that the results of this study are representative of FCM treatment efficacy in patients with iron deficiency in current clinical practice. Nevertheless, due to its retrospective and observational nature, this study presents some limitations. First, our analyses were based on the clinical and hematological data collected during routine clinical practice, which might have led to an apparent underreporting of adverse events and exclusion of some valid cases. Moreover, as several clinicians were involved in the prescription of FCM dosage, some patients were treated as recommended by the SmPC, while other patients were treated according to the Ganzoni formula. In addition, the lack of a clearly defined reference group limited our conclusions regarding the overall FCM treatment efficacy. Therefore, findings of this study, although valid, may only be considered as exploratory. Additional prospective studies should be performed, using appropriate populations and systematic documentation of hematological and TEAEs data. Future research should also address the efficacy and safety of intravenous FCM in different real-world clinical settings.

\section{Conclusions}

Our study showed that the use of FCM increases hemoglobin levels $\geq 2 \mathrm{~g} / \mathrm{dL}$ after 6 weeks in $41 \%$ of all iron deficiency patients, with or without anemia, in a hospital day setting and was associated with a low incidence of TEAEs. Moreover, treatment efficacy was significantly higher in patients who received higher cumulative FCM doses, that is, $501-1000$ and 1001-3000 mg, compared to patients who received doses of $500 \mathrm{mg}$. Our findings indicate that intravenous FCM dosage should be adjusted to improve treatment efficacy of iron deficiency in different clinical practice settings.

\section{Disclosure}

OM Pharma had no role in study design, or in collection, analysis, and interpretation of the data, or in the writing of the manuscript or the decision to submit the manuscript for publication.

\section{Conflicts of Interest}

António Robalo Nunes has received fees from OM Pharma for advice on intravenous ferric carboxymaltose treatment. Ana Palricas Costa, Sara Lemos Rocha, and Ana Garcia de Oliveira declare that there are no conflicts of interest regarding the publication of this article.

\section{Authors' Contributions}

António Robalo Nunes was responsible for study design. António Robalo Nunes, Ana Palricas Costa, Sara Lemos Rocha, and Ana Garcia de Oliveira were responsible for study conduct. António Robalo Nunes, Ana Palricas Costa, Sara Lemos Rocha, and Ana Garcia de Oliveira were responsible for data collection. António Robalo Nunes and Ana Palricas Costa were responsible for data analysis. António Robalo Nunes, Ana Palricas Costa, Sara Lemos Rocha, and Ana Garcia de Oliveira were responsible for data interpretation. António Robalo Nunes was responsible for drafting manuscript. António Robalo Nunes, Ana Palricas Costa, Sara Lemos Rocha, and Ana Garcia de Oliveira were responsible for revising manuscript content. António Robalo Nunes, Ana Palricas Costa, Sara Lemos Rocha, and Ana Garcia de Oliveira approved final version of manuscript.

\section{Acknowledgments}

OM Pharma provided a grant to support statistical analysis and medical writing services. The authors acknowledge Scientific Toolbox Consulting for providing consulting services in the following areas: statistical analysis and medical writing.

\section{References}

[1] E. McLean, M. Cogswell, I. Egli, D. Wojdyla, and B. De Benoist, "Worldwide prevalence of anaemia, WHO Vitamin and Mineral Nutrition Information System, 1993-2005," Public Health Nutrition, vol. 12, no. 4, pp. 444-454, 2009.

[2] World Health Organization, Iron Deficiency Anaemia: Assessment, Prevention and Control. A Guide for Programme Managers, World Health Organization, Geneva, Switzerland, 2001.

[3] C. Fonseca, F. Marques, A. Robalo Nunes, A. Belo, D. Brilhante, and J. Cortez, "Prevalence of anaemia and iron deficiency in Portugal: the EMPIRE study," Internal Medicine Journal, vol. 46, no. 4, pp. 470-478, 2016.

[4] C. Rognoni, S. Venturini, M. Meregaglia, M. Marmifero, and R. Tarricone, "Efficacy and Safety of Ferric Carboxymaltose and Other Formulations in Iron-Deficient Patients: A Systematic Review and Network Meta-analysis of Randomised Controlled 
Trials," Clinical Drug Investigation, vol. 36, no. 3, pp. 177-194, 2016.

[5] J. E. Toblli and M. Angerosa, "Optimizing iron delivery in the management of anemia: patient considerations and the role of ferric carboxymaltose," Drug Design, Development and Therapy, vol. 8, pp. 2475-2491, 2014.

[6] G. R. Bailie, N. A. Mason, and T. G. Valaoras, "Safety and tolerability of intravenous ferric carboxymaltose in patients with iron deficiency anemia," Hemodialysis International, vol. 14, no. 1, pp. 47-54, 2010.

[7] G. C, "Doron Schwartz, Should a search for iron deficiency be part of the regular screening in all patients, whether anemic or not?" Journal of Hematology \& Thromboembolic Diseases, vol. 02, no. 05, 2014.

[8] G. M. Keating, "Ferric carboxymaltose: a review of its use in iron deficiency," Drugs, vol. 75, no. 1, pp. 101-127, 2015.

[9] K. A. Lyseng-Williamson and G. M. Keating, "Ferric carboxymaltose: a review of its use in iron-deficiency anaemia," Drugs, vol. 69, no. 6, pp. 739-756, 2009.

[10] G. R. Bailie, "Efficacy and safety of ferric carboxymaltose in correcting iron-deficiency anemia: a review of randomized controlled trials across different indications.," ArzneimittelForschung, vol. 60, no. 6 a, pp. 386-398, 2010.

[11] J. E. Onken, D. B. Bregman, R. A. Harrington et al., "A multicenter, randomized, active-controlled study to investigate the efficacy and safety of intravenous ferric carboxymaltose in patients with iron deficiency anemia," Transfusion, vol. 54, no. 2, pp. 306-315, 2014.

[12] R. A. Moore, H. Gaskell, P. Rose, and J. Allan, "Meta-analysis of efficacy and safety of intravenous ferric carboxymaltose (Ferinject) from clinical trial reports and published trial data," BMC Blood Disorders, vol. 11, article 4, 2011.

[13] M. Kuster and D. N. Meli, "Treatment of iron deficiency with intravenous ferric carboxymaltose in general practice: a retrospective database study," Journal of Clinical Medicine Research, vol. 7, no. 1, pp. 37-40, 2015.

[14] M. Varcher, S. Zisimopoulou, O. Braillard, B. Favrat, and N. J. Perron, "Iron deficiency intravenous substitution in a Swiss academic primary care division: Analysis of practices," International Journal of General Medicine, vol. 9, pp. 221-227, 2016.

[15] L. Peyrin-Biroulet, N. Williet, and P. Cacoub, "Guidelines on the diagnosis and treatment of iron deficiency across indications: A systematic review," American Journal of Clinical Nutrition, vol. 102, no. 6, pp. 1585-1594, 2015.

[16] A. M. Ganzoni, "Intravenous iron-dextran: therapeutic and experimental possibilities," Schweizerische Medizinische Wochenschrift, vol. 100, no. 7, pp. 301-303, 1970.

[17] World Health Organization, International statistical classification of diseases and related health problems. - 10th revision, World Health Organization, Geneva, Switzerland, 2016.

[18] R. Mahey, A. Kriplani, K. D. Mogili, N. Bhatla, G. Kachhawa, and R. Saxena, "Randomized controlled trial comparing ferric carboxymaltose and iron sucrose for treatment of iron deficiency anemia due to abnormal uterine bleeding," International Journal of Gynecology and Obstetrics, vol. 133, no. 1, pp. 43-48, 2016.

[19] S. Garcia-Lopez, J. M. Bocos, J. P. Gisbert et al., "High-dose intravenous treatment in iron deficiency anaemia in inflammatory bowel disease: early efficacy and impact on quality of life," Blood Transfusion $=$ Trasfusione del Sangue, vol. 14, no. 2, pp. 199-205, 2016.
[20] M. Bach, T. Geisel, J. Martin et al., "Efficacy and Safety of Intravenous Ferric Carboxymaltose in Geriatric Inpatients at a German Tertiary University Teaching Hospital: A Retrospective Observational Cohort Study of Clinical Practice," Anemia, vol. 2015, Article ID 647930, 2015.

[21] R. Herfs, L. Fleitmann, and I. Kocsis, "Treatment of iron deficiency with or without anaemia with intravenous ferric carboxymaltose in gynaecological practices-a non-interventional study," Geburtshilfe und Frauenheilkunde, vol. 74, no. 1, pp. 8188, 2014.

[22] T. A. Koch, J. Myers, and L. T. Goodnough, "Intravenous Iron Therapy in Patients with Iron Deficiency Anemia: Dosing Considerations," Anemia, vol. 2015, Article ID 763576, 2015.

[23] B. Favrat, K. Balck, C. Breymann et al., "Evaluation of a single dose of ferric carboxymaltose in fatigued, iron-deficient women-PREFER a randomized, placebo-controlled study," PLoS ONE, vol. 9, no. 4, Article ID e94217, 2014.

[24] C. F. Barish, T. Koch, A. Butcher, D. Morris, and D. B. Bregman, "Safety and efficacy of intravenous ferric carboxymaltose $(750 \mathrm{mg})$ in the treatment of iron deficiency anemia: two randomized, controlled trials," Anemia, vol. 2012, Article ID 172104, 9 pages, 2012.

[25] P. Geisser and J. Banké-Bochita, "Pharmacokinetics, safety and tolerability of intravenous ferric carboxymaltose: a doseescalation study in volunteers with mild iron-deficiency anaemia," Arzneimittel-Forschung, vol. 60, no. 6a, pp. 362-372, 2010. 


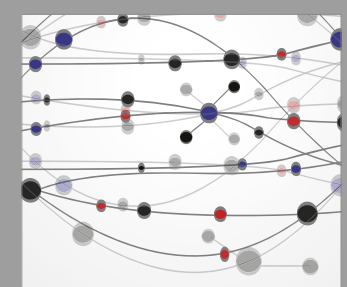

The Scientific World Journal
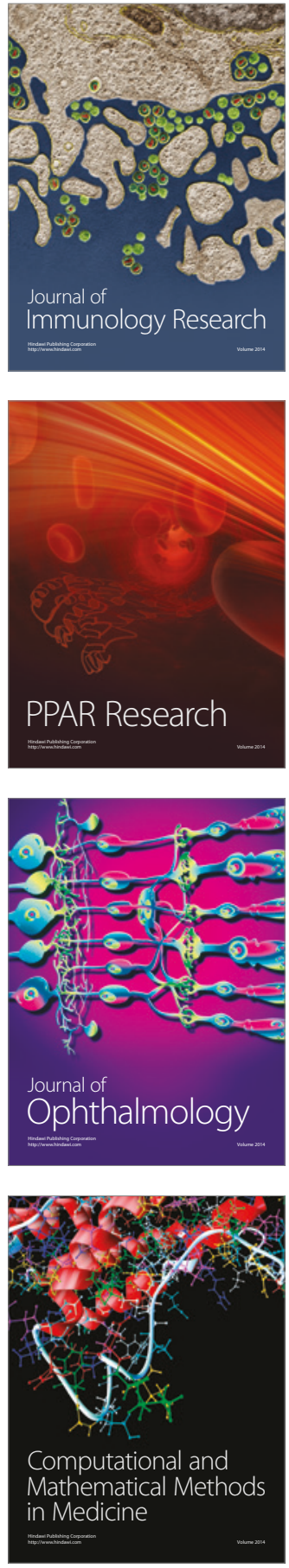

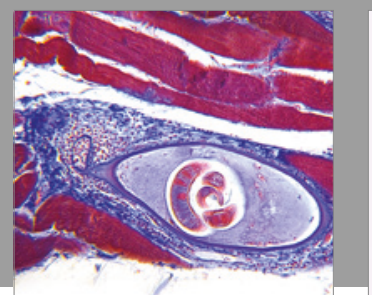

Gastroenterology Research and Practice
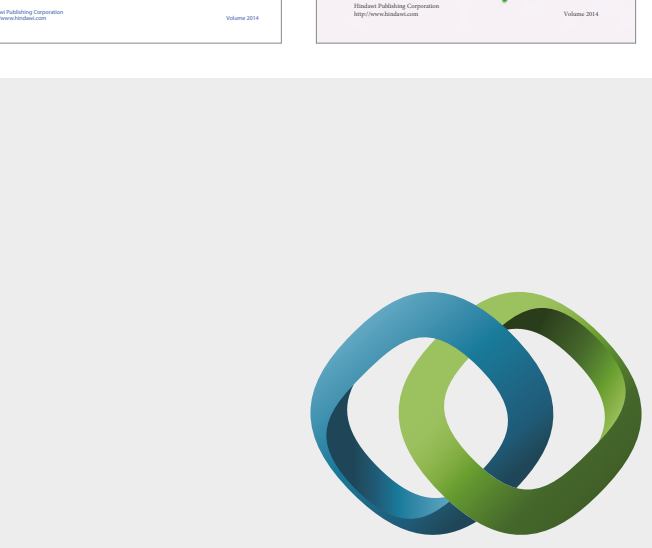

\section{Hindawi}

Submit your manuscripts at

https://www.hindawi.com
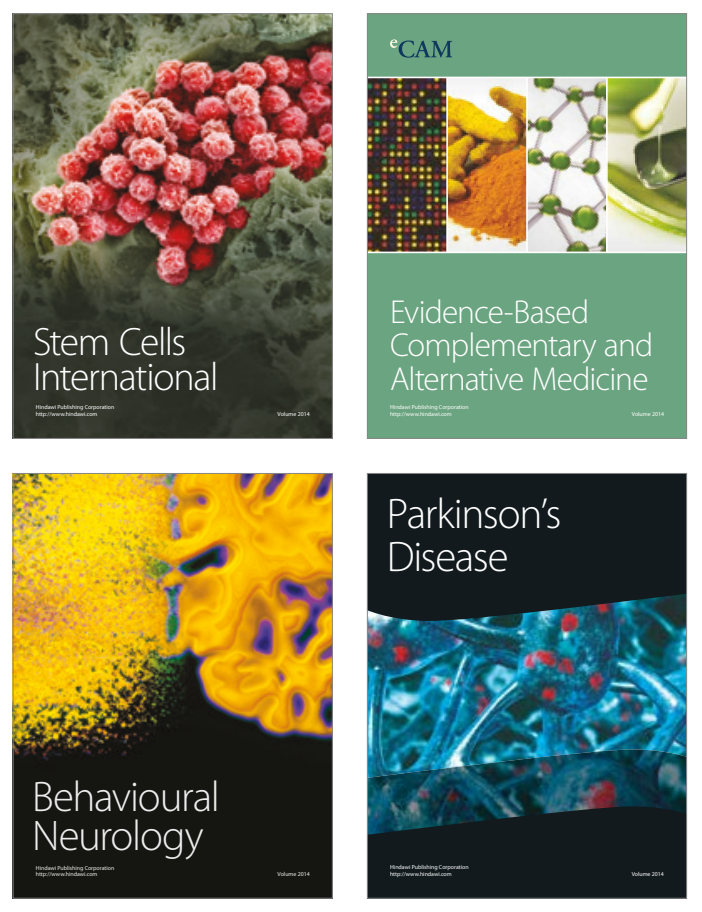
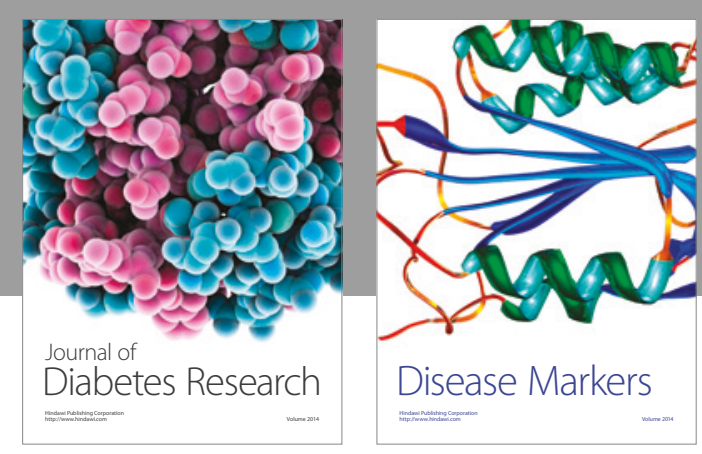

Disease Markers
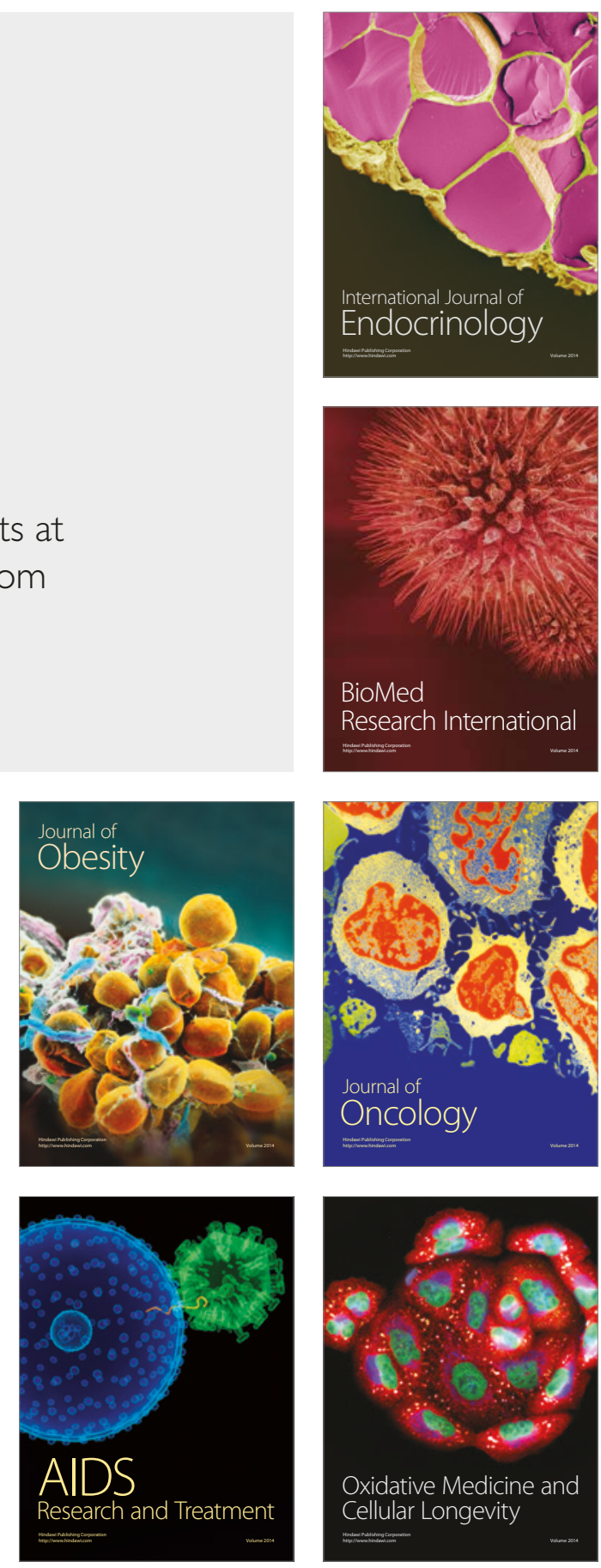